ment official I am discreet enough not to venture on a public discussion of the merits of so controversial a topic as the closure of the Moray Firth.

December $2 \mathrm{I}$. Henry G. Maurice.

\section{Propagation of a Finite Number of Waves.}

IT has been stated as a general proposition by physicists that it is impossible to propagate a finite number of simple harmonic waves without change of type, the reason being that the discontinuity of motion at the beginning and end of the train of waves cannot be represented by a simple sine or cosine function.

The proposition as stated is too general, and should be qualified. It would be true, however, to say that a finite number of simple harmonic waves cannot be propagated without change of type if the mean pressures in the wave-train and in the undisturbed medium are the same.

That a finite train of waves of unchanging type can actually be propagated in a medium in which the wave-velocity and group-velocity are the same may be seen from the following illustration:

Let the medium be enclosed in an indefinitely long pipe containing a piston on which a simple harmonic motion of amplitude $a$ may be imposed. Let the medium and piston be at rest when the latter is in one of the extreme positions. If the motion is then started there is no discontinuity either in the velocity or in the acceleration. So long as the motion of the piston is maintained, simple harmonic waves are propagated through the tube in both directions, the mean pressure in one set being $p_{0}(1+a / \lambda)$ and in the other $t_{0}(\mathrm{I}-a / \lambda)$, where $p_{0}$ is the undisturbed pressure.

At the moment when the motion is started the pressure on both sides of the piston is $p_{0}$, and this is the maximum or minimum pressure in each wave series, according as the direction of motion of the piston tends to dilate the medium or to compress it. The necessary increase and decrease of mean pressure in the two wave series are automatically supplied by the work done by the exterior force which must be applied to the piston to generate the successive waves. If the motion is stopped where the piston is in the same extreme position in which it was started, a finite number of simple harmonic waves are left travelling along the tube in opposite directions.

\section{A. Mallock.}

\section{Solar Variation and the Weather.}

Mr. Clayton's representation in Nature of December 9 that, apart from seasonal and diurnal changes, the "chief, if not the only," cause of weather change lies in solar variations overlooks an important source of variation in the atmosphere which could not be allowed for on the lines of his investigation. No one will dispute that if $\mathrm{Mr}$. Clayton finally substantiates his correlation results, which have been severely criticised in America by Dr. C. F. Marvin (Monthly Weather Review, March, I920) they will be of great value in forecasting deviations from the normal of particular elements, such as temperature and rainfall, in coming seasons. But they will not help us to foresee the actual sequence of atmospheric phases embodying the combination of all elements which we call weather; and it is the precise sequence of changes, not the departure of one or more elements from normal during a given period, which, after all, is the primary aim in weather forecasting.

It seems almost certain that however closely types may recur, the atmosphere is never twice in the same condition, and this, I believe, is a prominent conviction in the minds of those meteorologists with a lengthy experience of daily weather maps. If a series of phases in the circulation of the atmosphere be repre- sented by $A_{1} B_{1} C_{1} D_{1} \ldots$, it is conceivable that by an odd chance there might be a recurrence some time later of the particular phase $\mathrm{A}_{2}$; but unless, which seems almost infinitely improbable, all the external forces acting upon the atmosphere at the moment $A_{2}$ are identical with those which were in operation at the moment $A_{1}$, there will be no cyclic repetition $\mathrm{A}_{2} \mathrm{~B}_{2} \mathrm{C}_{2} \mathrm{D}_{2} \ldots$ to be maintained for a longer or shorter period. In place of the repeating cycle a very different series of phases might emerge; and it is on this principle that no tivo seasons are ever alike in the actual sequence of phases.

In other words, once set a fluid system like the earth's atmosphere in motion and change, it will possess an internal source of variability because identical dispositions of external forces never act upon identical phases. No doubt, with a sufficient number and quality of simultaneous observations, meteora logists could so diagnose the condition of the atmo sphere as to be able to calculate what phase would grow out of a present phase a few hours later; but the prevision of distant phases, which is really at the root of the weather problem, seems at present quite impossible. L. C. W. Bonacina.

27 Tanza Road, Hampstead, N.W.3,

December 19.

\section{Name for the Positive Nucleus.}

While the word "hydrion" does strictly express the meaning conveyed by the symbol $\mathrm{H}+$, yet in the minds of chemists it connotes all those properties associated with its hampered state in solutions, especially aqueous solutions. Even if hydrion is not hydrated like other ions, yet there is probably some sort of electrical double layer which modifies its properties. The "wet" physical chemist might abandon his term "hydrion" and the symbol, recognising that the real hydrion $(\mathrm{H}+)$ will have entirely different properties if ever these are known. He is in possession, however, and is not likely to do this unless, perhaps, some variant term, such as "hydro-ion," might be found acceptable. The alternative symbol $\mathrm{H}$ is, of course, already to hand, and, indeed, more widely used than $\mathrm{H}+$, which could be left to the physicists.

In spite of these minor difficulties, it appears to me that Prof. Soddy's suggestion (NATURE, December I6, p. 502) should be acceptable to chemists, largely because it is provisional in character and avoids the multiplication of words and symbols. Although it expresses a view as to the ultimate constitution of matter which may not prove correct, yet it is only the existence of such a view which necessitates a word and a symbol. If the other words suggested are to be taken as entirely non-committal, then the choice of one is less important at present, and, if effected, the word will probably soon acquire different meanings when used by different schools of thought.

E. B. R. Prideaux.

University College, Nottingham, December I8.

The Physical Meaning of Spherical Aberration.

MAY I correct an obvious mistake of mine which I notice in my letter on spherical aberration appearing in NATURE of December 9 ? The second expression for intensity should read :

$$
\begin{aligned}
\mathrm{I}=\mathrm{const} \times\left[\left\{\int_{a=0}^{a=a_{\mathrm{t}}} \mathrm{J}_{0}(a a) \cos \eta_{a} d\left(a^{2}\right)\right\}^{2}\right. & \\
& \left.+\left\{\int_{a=\infty}^{a=\mathrm{s}_{\mathrm{\tau}}} \mathrm{J}_{0}(a a) \sin \eta_{a} d\left(a^{2}\right)\right\}\right] \\
& \text { L. C. MARTIN. }
\end{aligned}
$$

Imperial College, S.W.7, December I7. No. 2670 , VOL. I06] 